\title{
Fonsecaea pedrosoi: A rare cause of dental infection and maxillary osteomyelitis in a child with acute lymphoblastic leukemia
}

\author{
Tuğçe Tural-Kara ${ }^{1}$, Halil Özdemir ${ }^{1}$, Elif İnce ${ }^{2}$, Talia İleri ${ }^{2}$, Ergin Çiftçi ${ }^{1}$ \\ Divisions of ${ }^{1}$ Pediatric Infectious Diseases and ${ }^{2}$ Pediatric Hematology and Oncology, Department of Pediatrics, Ankara \\ University Faculty of Medicine, Ankara, Turkey.E-mail: tugcetural@hotmail.com \\ Received: 19th April 2016, Revised: 6th May 2016, Accepted: 10th June 2016
}

\begin{abstract}
SUMMARY: Tural-Kara T, Özdemir H, İnce E, İleri T, Çiftçi E. Fonsecaea pedrosoi: A rare cause of dental infection and maxillary osteomyelitis in a child with acute lymphoblastic leukemia. Turk J Pediatr 2016; 58: 679-682.

Dental lesions are commonly seen in children with malignancy. We report a child with acute lymphoblastic leukemia who had black-brown dental lesion during the febrile neutropenic episode. Histopathological examination of dental lesion showed fungal hyphae and conidia. F. pedrosoi that was isolated from the tissue culture. The patient was treated with intravenous liposomal amphotericin B therapy for 5 weeks and he was discharged on oral voriconazole. On follow-up, clinical symptoms recovered. Although $F$. pedrosoi may be an unusual causative agent of dental infection and maxillary osteomyelitis, it should be considered in patient with black-brown lesions which do not respond to antibacterial treatment.
\end{abstract}

Key words: acute lymphoblastic leukemia, children, dental infection, Fonsecaea pedrosoi, osteomyelitis.

Patients with acute myeloid leukemia, relapsed leukemia, those receiving highly myelosuppressive chemotherapy and allogeneic hematopoietic stem cell transplant recipients have high risk for fungal infections. Even though invasive fungal infection related mortality can be reduced with prophylactic antifungal therapy, it still continues to be a major cause of morbidity and mortality in neutropenic patients ${ }^{1}$. The incidence of fungal infections in acute lymphoblastic leukemia (ALL) is reportedly $24 \%$ and it can increase with prolonged duration of neutropenia ${ }^{2}$.

Chromoblastomycosis per se, is an infection of skin and subcutaneous tissue ${ }^{3}$. Chromoblastomycosis is a well-established disease caused by F. pedrosoi and have just a few case reports. However, F. pedrosoi associated dental infections and maxillary osteomyelitis have not been reported. To our knowledge, this is the first reported pediatric case of dental infection and maxillary osteomyelitis caused by F. pedrosoi.

\section{Case Report}

A 6-year-old boy with a diagnosis of standard risk B precursor ALL presented with fever on the $43^{\text {rd }}$ day of delayed intensification therapy as per COG AALL0331 chemotherapy regimen. On physical examination, he was febrile and pale. His vital signs included a temperature of $39{ }^{\circ} \mathrm{C}$, heart rate of 128 beats per minute, respiratory rate of 32 breaths per minute, and a blood pressure of $86 / 52 \mathrm{mmHg}$. He had $3 \mathrm{~cm}$ hepatomegaly below the right costal margin and $2 \mathrm{~cm}$ splenomegaly below the left costal margin. The rest of physical examination was normal, and no infectious focus was detected. Patient's laboratory test results were as followed; hemoglobin 11.7 $\mathrm{g} / \mathrm{dl}$, white blood cell count $0.2 \times 10^{3} / \mathrm{mm}^{3}$, absolute neutrophil count $0 / \mathrm{mm}^{3}$ (neutrophils $0 \%$, lymphocytes $90 \%$, monocytes $4 \%$ and eosinophils $6 \%$ ), platelet count $16 \times 10^{3} / \mathrm{mm}^{3}$, C-reactive protein $62 \mathrm{mg} / \mathrm{L}$. He was started on intravenous cefoperazone-sulbactam for fever and neutropenia. The following day, intravenous teicoplanin and acyclovir were 

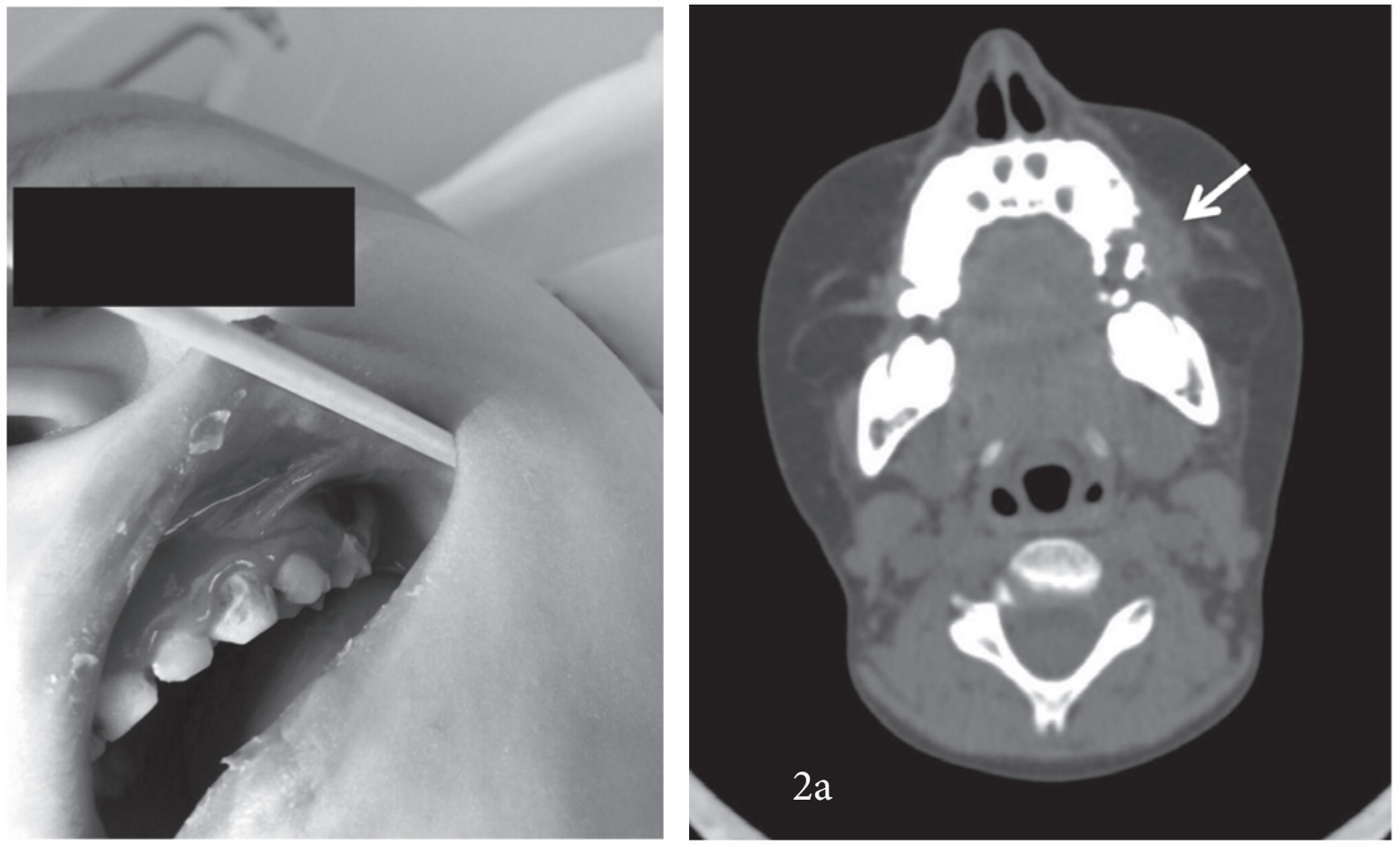

Fig. 1. Necrotic lesion near the upper left molar tooth
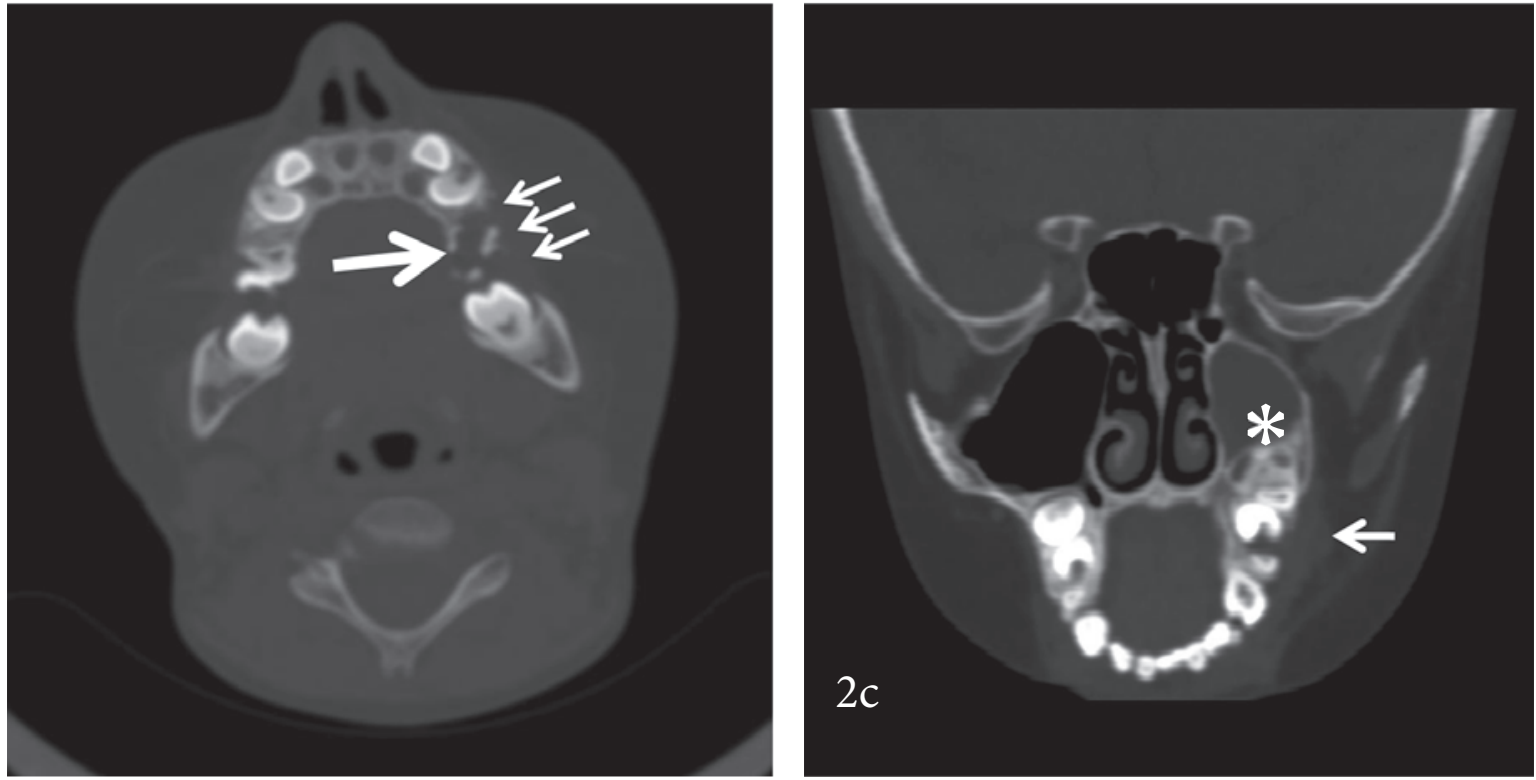

Fig. 2. Axial CT scans, soft tissue (arrow) is seen on the left side of maxilla; (a) bone windowing (b) clearly shows dental loss (large arrow), cortical discontuinity of maxillary bone (small arrows) (c) cortical destruction and soft tissue density (arrow), compatible with osteomyelitis are better seen on coronal reformatted CT image. Also note left maxillary sinusitis (asterisk).

added to the treatment because of mucositis and vesicular lesions on his upper lip. On the $5^{\text {th }}$ day of treatment, cefoperazone-sulbactam was discontinued and intravenous meropenem and liposomal amphotericin B therapy was started due to the ongoing persistent fever.

On follow-up, necrotic areas were noted near the upper left molar tooth (Fig. 1). Computed tomography of the paranasal sinuses showed soft tissue density and sinusitis on the left 

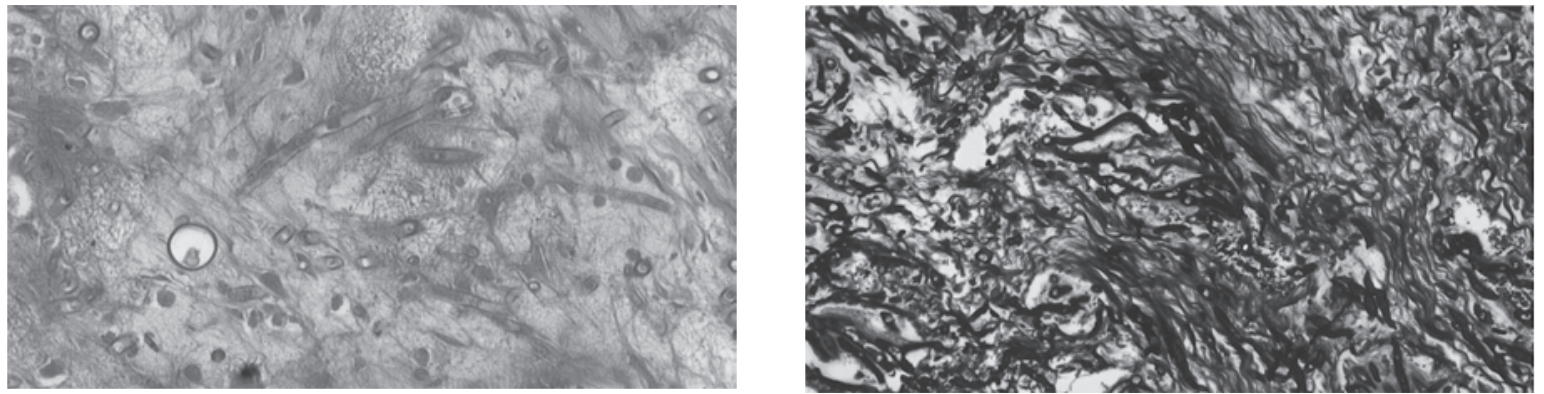

Fig. 3a, 3b. Fungal branching septate hyphae and conidia on the hematoxylin-eosin and Grocott's methenamine silver stained

side of maxilla, dentin loss of the upper left second premolar tooth, and cortical destruction of maxillary bone which was considered as osteomyelitis (Fig. 2a, 2b, 2c). With the possibility of invasive fungal infection, the tooth was pulled. Histopathological examination showed fungal hyphae and conidia on the hematoxylin-eosin and Grocott's Methenamine Silver stained section (Fig. $3 \mathrm{a}, 3 \mathrm{~b}$ ) and $F$. pedrosoi was isolated from the tissue culture. The patient received intravenous liposomal amphotericin B therapy for 5 weeks and he was discharged on oral voriconazole. His complaints decreased markedly. Currently at the $7^{\text {th }}$ month of treatment because of the possible risk for prolonged neutropenia, the patient remains on oral voriconazole therapy without any adverse effects.

\section{Discussion}

Acute lymphoid leukemia is a common pediatric malignancy. Fungal infections are a major problem in neutropenic children ${ }^{1}$.

Fonsecaea is a fungal species in Herpotrichiellaceae family and it is a dermatiaceous and filamentous fungus. The genus Fonsecaea has three species: F. pedrosoi, F. monophora and F. nubica. F. pedrosoi is mostly found in tropical and subtropical regions. It can cause infection by the invasion of hypha or conidia in damaged tissues ${ }^{4}$. In our case there was no major trauma reported by the parents and the patient, but the microtrauma during tooth brushing was thought to be the possible reason for the infection.

F. pedrosoi has dark brown hyphae, erect conidiophores and brown, barrel-shaped conidia. The important distinguishing feature is melanin in the cell wall and it causes appearance of black-brown lesions ${ }^{5}$. In our patient a black necrotic lesion was seen on the gingiva.
F. pedrosoi associated chromoblastomycosis have been often reported in the literature furthermore cerebral granuloma, brain abscess, keratitis and septic arthritis which are secondary to $F$. pedrosoi have been published ${ }^{6-9}$.

Fungal osteomyelitis is rare and there are few cases of $F$. pedrosoi osteomyelitis in the literature ${ }^{9,10}$. Karuppal et al. ${ }^{10}$ have reported a 50-year-old immunocompetent male who had pain, swelling and multiple discharging sinuses on the left ankle for 3 years. Computed tomography imaging showed lytic areas on the left tibia. Then tibial osteomyelitis was diagnosed secondary to $F$. pedrosoi which was isolated from the tissue culture. The patient was successfully treated with itraconazol and surgical debridement. In addition, De Guzman et al. ${ }^{9}$ have reported $F$. pedrosoi osteomyelitis secondary to chromoblastomycosis. Likewise the patient completely recovered with antifungal therapy.

Although standard treatment approach has not been established, surgical excision and antifungal medications are recommended for treatment. Posaconazole, itraconazol, amphotericin $\mathrm{B}$, voriconazol and flucytosine are found to be effective. However, echinocandins are not recommended for treatment due to the high minimum inhibitory concentration (MIC) levels required for effective treatment and to the lack of oral formulations ${ }^{11}$. There is no clear data on the duration of treatment but the general recommendation is not to discontinue the treatment too early and repeat biopsy might be necessary to show the negative results on the direct microscopic examination and the fungal cultures in some cases ${ }^{12}$.

The mouth has been documented as the most common source of sepsis in immunosuppressed patients. Oral manifestations like gingivitis, 
mucositis, herpetic lesions, bleeding, erythema, petechia, ulceration and candidiasis are frequently reported in patients with $\mathrm{ALL}^{13}$. However, there are no cases reported with F. pedrosoi associated dental infections and maxillary osteomyelitis in the literature.

As a result, oral lesions may be seen in patients during neutropenic period and F. pedrosoi is an unusual causative agent of dental infection and maxillary osteomyelitis. However, it should be considered in the differential diagnosis of black-brown lesions which do not respond to antibacterial therapy in neutropenic patients with malignancy.

\section{REFERENCES}

1. Hahn-Ast C, Glasmacher A, Mückter S, et al. Overall survival and fungal infection-related mortality in patients with invasive fungal infection and neutropenia after myelosuppressive chemotherapy in a tertiary care centre from 1995 to 2006. J Antimicrob Chemother 2010; 65: 761-768.

2. Sahbudak Bal Z, Yilmaz Karapinar D, Karadas N, et al. Proven and probable invasive fungal infections in children with acute lymphoblastic leukaemia: results from an university hospital, 2005-2013. Mycoses 2015; 58: $225-232$

3. Alviano DS, Franzen AJ, Travassos LR, et al. Melanin from Fonsecaea pedrosoi induces production of human antifungal antibodies and enhances the antimicrobial efficacy of phagocytes. Infect Immun 2004; 72: 229-237.

4. Chaidaroon W, Tananuvat $\mathrm{N}$, Chavengsaksongkram P, Vanittanakom N. Corneal chromoblastomycosis caused by Fonsecaea pedrosoi. Case Rep Ophthalmol 2015; 27: 82-87. 5. Santos AL, Palmeira VF, Rozental S, et al. Biology and pathogenesis of Fonsecaea pedrosoi, the major etiologic agent of chromoblastomycosis. FEMS Microbiol Rev 2007; 31: 570-591.
6. Madhugiri VS, Bhagavatula ID, Mahadevan A, Siddaiah $\mathrm{N}$. An unusual infection, an unusual outcome-Fonsecaea pedrosoi cerebral granuloma. J Neurosurg Pediatr 2011; 8: 229- 232

7. Madhugiri VS, Singh R, Vyavahare $M$, et al. Opportunistic Fonsecaea pedrosoi brain abscess in a patient with non-cirrhotic portal fibrosis-induced hypersplenism--a novel association. Br J Neurosurg 2013; 27: 690-693.

8. Sangwan J, Lathwal S, Juyal D, Sharma N. Fonsecaea pedrosoi: a rare etiology in fungal keratitis. J Clin Diagn Res 2013; 7: 2272-2273.

9. De Guzman L, Perlman DC, Hubbard CE. Septic arthritis and osteomyelitis due to the chromoblastomycosis agent Fonsecaea pedrosoi. Am J Orthop (Belle Mead NJ) 2012; 41: 328-331.

10. Karuppal R, Kumaran CM, Marthya A, et al. Tibial osteomyelitis due to Fonsecaea pedrosoi in an immunocompetent patient: case report. J Foot Ankle Surg 2009; 48: 569-572.

11. Najafzadeh MJ, Badali H, Illnait-Zaragozi MT, De Hoog GS, Meis JF. In vitro activities of eight antifungal drugs against 55 clinical isolates of Fonsecaea spp. Antimicrob Agents Chemother 2010; 54: 1636-1638.

12. Poirriez J, Breuillard F, Francois N, et al. A case of chromomycosis treated by a combination of cryotherapy, shaving, oral 5-flurocytosine, and oral amphotericin B. Am J Trop Med Hyg 2000; 63: 61-63.

13. Ponce-Torres E, Ruíz-Rodríguez Mdel S, AlejoGonzález F, Hernández-Sierra JF, Pozos-Guillén Ade $\mathrm{J}$. Oral manifestations in pediatric patients receiving chemotherapy for acute lymphoblastic leukemia. J Clin Pediatr Dent 2010; 34: 275-279. 